\title{
SHEAVES ON SINGULAR VARIETIES
}

\author{
ELIZABETH GASPARIM AND THOMAS KÖPPE
}

\begin{abstract}
We prove existence of reflexive sheaves on singular surfaces and threefolds with prescribed numerical invariants and study their moduli.
\end{abstract}

\section{Motivation}

Sheaves on singular varieties have become very popular recently because of their appearance in Physics, String Theory and Mirror Symmetry. In particular, many open questions about sheaves on singular varieties have come to light. The corresponding mathematical tools, however, are waiting to be developed. Our aim in this paper is to entice singularists to develop some basic techniques needed to approach such questions.

It is extremely common for a physicist or string theorist to start up a lecture by giving a partition function for a theory, and now even algebraic geometers are quite often doing the same. It is not just a fashion, but the fact is that this is an extremely efficient way to present results. The general format of such partition functions is of an infinite sum whose terms contain integrals over moduli spaces. Here are some examples. We will not need details from these expressions, just the observation that they all contain integrals over moduli spaces.

Example 1.1. (String Theory) The Nekrasov partition function for $N=2$ supersymmetric $S U(r)$ pure gauge theory on a complex surface $X$ is given by an expression of the form

$$
Z_{X}:=\Lambda^{(1-r) d \cdot d} \sum_{n \geq 0} \Lambda^{2 r n} \int_{\mathfrak{M}_{r, d, n}(X)} 1,
$$

where $\mathfrak{M}_{r, d, n}(X)$ is the moduli space of framed torsion-free sheaves or rank $r$, and Chern classes $c_{1}=d$ and $c_{2}=n$. For the case of gauge theories with matter, one writes a similar expression but with more interesting integrands, see [GL].

Example 1.2. (Donaldson-Thomas Theory) For a Calabi-Yau threefold $X$, the partition function for Donaldson-Thomas theory is given by:

$$
Z_{X}:=\sum_{\beta \in H_{2}(X, \mathbb{Z})} \sum_{n \in \mathbb{Z}} Q^{n} v^{\beta} \int_{\left[I_{n}(X, \beta)\right]^{\mathrm{vir}}} 1
$$

where $I_{n}(X, \beta)$ is the moduli space of ideal sheaves $\mathcal{I}$ fitting into an exact sequence

$$
0 \longrightarrow \mathcal{I} \longrightarrow \mathcal{O}_{X} \longrightarrow \mathcal{O}_{Y} \longrightarrow 0
$$

and satisfying

$$
\chi\left(\mathcal{O}_{Y}\right)=n
$$

and $[Y]=\beta \in H_{2}(X, \mathbb{Z})$, where $\chi$ is the holomorphic Euler characteristic, see [MNOP].

Key words and phrases. Reflexive sheaves, local homolorphic Euler characteristic, moduli spaces. 
Example 1.3. (Gromov-Witten Theory) For a Calabi-Yau threefold $X$, the partition function for degree- $\beta$ Gromov-Witten invariants is given by

$$
Z_{X}:=\exp \sum_{\beta \neq 0} \sum_{g \geq 0} u^{2 g-2} v^{\beta} \int_{\left[\overline{M_{g}}(X, \beta)\right]^{\mathrm{vir}}} 1,
$$

where $\overline{M_{g}}(X, \beta)$ is the moduli space of genus- $g$ curves representing the class $\beta \in H_{2}(X, \mathbb{Z})$. There is a precise sense in which this partition function is equivalent to the one in Example 1.2, see $[\mathrm{MNOP}]$.

These examples illustrate the appearance of integrals over moduli spaces of sheaves. Even in the case of moduli spaces of maps of Example 1.3 the theory is still related to a theory given by integration over moduli of sheaves. Observe that the definition of moduli spaces itself requires a choice of numerical invariants: in Example 1.1 the Chern classes and in Example 1.2 the Euler characteristic. So, we now agree that we are interested in moduli spaces of sheaves on surfaces and threefolds. Of course, the physics motivation is just a bonus, and we could have been interested in such moduli spaces for purely geometric reasons, as they are part of classical algebraic geometry. Now physics dictates that we should consider theories defined over singular varieties. In fact, some of the most popular categories considered currently by physicists and string theorists turn out empty in the absence of singularities; such is the case of the FukayaSeidel category and the Orlov category of singularities. Thus we arrive at the conclusion that we need to understand moduli of sheaves on singular varieties. Both the case of global moduli of sheaves on projective varieties and the case of local moduli on a small neighborhood of a singularity are of interest. For the local case there is an added difficulty: What are the correct numerical invariants to be considered? In this paper we will show that the local holomorphic Euler characteristic provides a satisfactory invariant for sheaves on local surfaces. For the case of local threefolds however, the study of numerical invariants is work in progress, and much remains to be done. The goal of this paper is to describe partial progress in the understanding of these questions. We define new numerical invariants for the threefold case, and give existence of sheaves with given local numerical data.

\section{ACKNOWLEDGEMENTS}

We are very happy to contribute to this volume in honour of Andrew DuPlessis, and thankful to Christophe Eyral for giving us this opportunity. The final version of this article was completed while the first author visited UNICAMP under FAPESP project number 2009/08587-5; their hospitality and generous support is hereby gladly acknowledged.

\section{Main Results}

In this paper we consider rational surface singularities obtained by contracting a line $\ell \cong$ $\mathbb{P}^{1}$ inside a smooth surface or threefold. Numerous approaches using numerical invariants or characteristic classes of some sort have been proposed in the past, see e.g. [Br], and in Section 3 we define numerical invariants, some of which are new, that we need for the present situation. To set the stage, in Section 4 we recall some of our earlier results for sheaves on singular surfaces. The results for threefolds presented in Section 5 are new and will appear in more detail in [Kö].

In Section 3 we define the local holomorphic Euler characteristic $\chi(\ell, \mathcal{F})$ of a reflexive sheaf $\mathcal{F}$. We will present the following results.

Theorem 4.4. Let $\mathfrak{M}_{n}\left(X_{k}\right)$ be the moduli of reflexive sheaves on $\mathbb{C}^{2} / \mathbb{Z}_{k}$ with local holomorphic Euler characteristic equal to $n$. Then for all $n \geq 0, \mathfrak{M}_{n}\left(X_{k}\right)$ is non-empty. 
Theorem 5.1. For every rank-2 bundle $E$ on $W_{1}:=\operatorname{Tot}\left(\mathcal{O}_{\mathbb{P}^{1}}(-1) \oplus \mathcal{O}_{\mathbb{P}^{1}}(-1)\right)$ with $c_{1}(E)=0$ and $\left.E\right|_{\mathbb{P}^{1}} \cong \mathcal{O}_{\mathbb{P}^{1}}(-j) \oplus \mathcal{O}_{\mathbb{P}^{1}}(j)$, the following bounds are sharp:

$$
j-1 \leq \chi\left(\ell, \pi_{*} E\right)=\mathbf{h}(E) \leq\left(j^{2}+j\right)(j-1) / 6 .
$$

Here $\pi: W_{1} \rightarrow X$ is the contraction of the zero section $\ell$ and $X$ is the singular threefold $x y-z w=0$ in $\mathbb{C}^{4}$.

Theorem 5.7. Let $X$ be the singular threefold $x y-z w=0$ in $\mathbb{C}^{4}$. For each $j \geq 2$ there exists $a(4 j-5)$-dimensional family of rank-2 reflexive sheaves on $X$ with local holomorphic Euler characteristic $j-1$.

For each of the cases $j=0$ or 1 , our methods produce only the direct images of the split sheaves; both have local holomorphic Euler characteristic 0.

\section{Numerical InVARIANTS}

In this section we define numerical invariants for sheaves on a neighborhood of a singularity. Our first invariant is defined for any dimension, and is particularly adapted to study reflexive sheaves that are direct images of vector bundles on a resolution. Let $\pi:(Z, \ell) \rightarrow(X, x)$ be a resolution of an isolated quotient singularity, $\mathcal{F}$ a reflexive sheaf on $Z$ and $n:=\operatorname{dim} X$. The following definition is due to Blache, [Bl, Def. 3.9].

Definition 3.1. The local holomorphic Euler characteristic of $\pi_{*} \mathcal{F}$ at $x$ is

$$
\chi\left(x, \pi_{*} \mathcal{F}\right):=\chi(\ell, \mathcal{F}):=h^{0}\left(X ;\left(\pi_{*} \mathcal{F}\right)^{\vee \vee} / \pi_{*} \mathcal{F}\right)+\sum_{i=1}^{n-1}(-1)^{i-1} h^{0}\left(X ; R^{i} \pi_{*} \mathcal{F}\right) .
$$

For the case when $X$ is a compact orbifold, Blache [Bl] shows that the global Euler characteristics of $X$ and its resolution are related by

$$
\chi\left(X,\left(\pi_{*} \mathcal{F}\right)^{\vee \vee}\right)=\chi(Z, \mathcal{F})+\sum_{x \in \operatorname{Sing} X} \chi\left(x, \pi_{*} \mathcal{F}\right) .
$$

Example 3.2. (Homological dimension 1) Consider the case when $Z$ is itself the total space of a bundle on $\mathbb{P}^{1}$. Then $Z$ has homological dimension one, and the expression on the right-hand side of (3.1) reduces to two terms, which we call the width and height of $\mathcal{F}$, respectively:

$$
\mathbf{w}(\mathcal{F}):=h^{0}\left(X ;\left(\pi_{*} \mathcal{F}\right)^{\vee \vee} / \pi_{*} \mathcal{F}\right) \quad \text { and } \quad \mathbf{h}(\mathcal{F}):=h^{0}\left(X ; R^{1} \pi_{*} \mathcal{F}\right) .
$$

Hence $\chi=\mathbf{w}+\mathbf{h}$.

The case when $Z$ is the total space of a negative line bundle on $\mathbb{P}^{1}$ was studied in [BGK1] and $[\mathrm{GKM}]$. Unfortunately, the width vanishes in higher dimensions.

Lemma 3.3. [BGK1, Lemma 5.2] Let $C$ be a curve of codimension $n \geq 2$ in $Z$ and $\pi: Z \rightarrow X$ the contraction of $C$ to a point. Then for any reflexive sheaf $\mathcal{F}$ on $Z$ we have

$$
h^{0}\left(X ;\left(\pi_{*} \mathcal{F}\right)^{\vee \vee} / \pi_{*} \mathcal{F}\right)=0 \text {. }
$$

Example 3.4. (Flop) When $W_{1}=\operatorname{Tot}\left(\mathcal{O}_{\mathbb{P}^{1}}(-1) \oplus \mathcal{O}_{\mathbb{P}^{1}}(-1)\right)$, Lemma 3.3 shows that $\mathbf{w}=0$. The height is still a non-trivial invariant, but less powerful than on surfaces.

However, we can define new invariants by restricting to sub-surfaces. We have two divisors $\left.D_{0}:=\operatorname{Tot}\left(\mathcal{O}_{\mathbb{P}^{1}}(-1) \oplus\{0\}\right)\right)$ and $D_{\infty}:=\operatorname{Tot}\left(\{0\} \oplus \mathcal{O}_{\mathbb{P}^{1}}(-1)\right)$, which are both isomorphic to $Z_{1}$, and they span the linear system

$$
|D|:=\left\{\lambda_{0} D_{0}+\lambda_{\infty} D_{\infty}:\left[\lambda_{0}: \lambda_{\infty}\right] \in \mathbb{P}^{1}\right\} .
$$

Then each $D_{\lambda} \in|D|$ is isomorphic to $Z_{1}$, and by restriction to $D_{\lambda}$ we can define an entire family of pairs $(\mathbf{w}, \mathbf{h})$. 
We now return to the case when $Z$ is the total space of a vector bundle over $\ell=\mathbb{P}^{1}$ and there is a contraction $\pi: Z \rightarrow X$. We will construct sheaves on $X$ as direct images of bundles on $Z$, which we now describe. For simplicity, we consider rank-2 bundles with vanishing $c_{1}$. The general case is no more difficult, but more unwieldy to present. When $\left.E\right|_{\ell} \cong \mathcal{O}_{\mathbb{P}^{1}}(-j) \oplus \mathcal{O}_{\mathbb{P}^{1}}(j)$, we call the integer $j \geq 0$ the splitting type of $E$. It turns out that the ampleness of the conormal bundle of $\ell$ implies that $E$ is an algebraic extension of line bundles,

$$
0 \longrightarrow \mathcal{O}(-j) \longrightarrow E \longrightarrow \mathcal{O}(j) \longrightarrow 0 \text {. }
$$

A line bundle $\mathcal{O}(n)$ is uniquely determined as the pullback of $\mathcal{O}_{\mathbb{P}^{1}}(n)$ from $\mathbb{P}^{1}$, since $\operatorname{Pic} Z \cong$ $\operatorname{Pic} \mathbb{P}^{1}$. For every $j \geq 0$, there is the trivial extension $\mathcal{O}(-j) \oplus \mathcal{O}(j)$, which we call the split bundle of splitting type $j$. For convenience, we sometimes write $E_{\text {split }}$ for the split bundle of the same splitting type as a given bundle $E$.

The first cohomology of $\mathcal{E} n d E$ is finite-dimensional and furnishes us with our next invariant:

$$
h^{1}(Z ; \mathcal{E} n d E)
$$

Naturally, we wish to consider the zeroth cohomology as well. Sadly, this is infinite-dimensional, so extra effort is required. We consider the $m^{\text {th }}$ infinitesimal neighbourhood of $\ell$, denoted $\ell^{(m)}$, which is a projective scheme. The restriction $E^{(m)}:=\left.E\right|_{\ell^{(m)}}$ is coherent. For $i=0,1$, we set

$$
\psi_{m}^{i}(E):=h^{i}\left(\ell^{(m)} ; E^{(m)}\right),
$$

thus $\psi_{m}^{i}$ takes finite values. We find that the difference $\psi_{m}^{i}\left(E_{\text {split }}\right)-\psi_{m}^{i}(E)$ is eventually constant.

Definition 3.5. For $i=0,1$ and $m \gg 0$, set

$$
\Delta_{i}(E):=\psi_{m}^{i}\left(E_{\text {split }}\right)-\psi_{m}^{i}(E) \text {. }
$$

For $h^{1}$, of course, this step is needlessly complicated, as the first cohomology is actually finite-dimensional, but this way the method may be applied to spaces in which the conormal bundle of $\ell$ is not ample.

The two numbers $\Delta_{0}$ and $\Delta_{1}$ are related via the Hilbert polynomial. Recall that for any coherent sheaf $\mathcal{A}$ on a projective scheme $S$, the Hilbert series

$$
\phi(\mathcal{A}, n):=\chi(\mathcal{A}(n)):=\sum_{i \geq 0}(-1)^{i} h^{i}(S ; \mathcal{A}(n))
$$

is a polynomial of degree $\operatorname{dim} S$. We have

$$
\Delta_{0}(E)-\Delta_{1}(E)=\phi\left(E^{(m)}, 0\right)-\phi\left(E_{\text {split }}^{(m)}, 0\right) .
$$

But the Hilbert polynomials of $E^{(m)}$ and $E_{\text {split }}^{(m)}$ are the same, as we will show momentarily, and so we have $\Delta_{0}=\Delta_{1}$, and for computational ease we just stick with $h^{1}(\mathcal{E} n d E)$. The equality of the Hilbert polynomials, and consequently the fact that the Hilbert polynomial does not see the extension (3.4), is a consequence of the following result.

Lemma 3.6. Let $E$ be an extension of type (3.4) with splitting type $j$ on either $Z_{k}:=\operatorname{Tot}\left(\mathcal{O}_{\mathbb{P}^{1}}(-k)\right)$ or $W_{1}:=\operatorname{Tot}\left(\mathcal{O}_{\mathbb{P}^{1}}(-1) \oplus \mathcal{O}_{\mathbb{P}^{1}}(-1)\right)$. Then the Hilbert polynomial of $\left.E\right|_{\ell^{m}}$,

$$
\begin{aligned}
\phi\left(E^{(m)}, n\right) & =\chi\left(E^{(m)}(n)\right) \\
& :=\sum_{i}(-1)^{i} h^{i}\left(\ell^{(m)} ;\left.E(n)\right|_{\ell^{(m)}}\right)= \begin{cases}(m+1)(k m+2+2 n) & \text { on } Z_{k}, \\
\frac{1}{3}(m+2)(m+1)(2 m+3 n+3) & \text { on } W_{1},\end{cases}
\end{aligned}
$$

is independent of the extension class, and independent of the splitting type $j$. Similarly, the Hilbert polynomial of the endomorphism bundle $\left.\mathcal{E} n d E\right|_{\ell^{(m)}}$ is $2 \phi\left(E^{(m)}, n\right)$. 


\begin{tabular}{ccccccc}
\hline & $w(E)$ & $h(E)$ & $h^{1}(\mathcal{E} n d E)$ & $w(G)$ & $h(G)$ & $h^{1}(\mathcal{E} n d G)$ \\
\hline$Z_{1}$ & 6 & 3 & 15 & 1 & 2 & 9 \\
$Z_{2}$ & 2 & 2 & 9 & 0 & 2 & 7 \\
$Z_{3}$ & 1 & 2 & 7 & 0 & 2 & 6 \\
$W_{1}$ & 0 & 4 & 35 & 0 & 2 & 17 \\
\hline
\end{tabular}

TABLE 1. The invariants width, height and $h^{1}(\mathcal{E} n d)$ for the split bundle $E$ and a generic bundle $G$ of splitting type $j=3$ on the spaces $Z_{1}, Z_{2}, Z_{3}$ and $W_{1}$.

Proof. By the additivity of the Hilbert polynomial on short exact sequences, the Hilbert polynomials in question are determined by the Hilbert polynomial of the line bundles $\mathcal{O}_{\ell^{(m)}}(p)$ for all $p$. Since $\mathcal{O}_{\ell^{(m)}}(1)$ is ample, the higher cohomology of $\mathcal{O}_{\ell^{(m)}}(p)$ vanishes for sufficiently large $p$. (We can verify this by direct computation.)

Being a polynomial, the Hilbert polynomial is determined by finitely many values, so it suffices to compute $\phi\left(\mathcal{E} n d E^{(m)}, n\right)=h^{0}\left(\ell^{(m)} ; \mathcal{O}_{\ell^{(m)}}(p)\right)$ for large $p$. Since $E$ and $\mathcal{E} n d E$ have filtrations by line bundles, which restrict to filtrations on every infinitesimal neighbourhood $\ell^{(m)}$, we compute:

$$
\begin{aligned}
\phi\left(E^{(m)}, n\right) & =\phi\left(\mathcal{O}_{\ell^{(m)}}(-j), n\right)+\phi\left(\mathcal{O}_{\ell^{(m)}}(j), n\right), \text { and } \\
\phi\left(\mathcal{E} n d E^{(m)}, n\right) & =\phi\left(\mathcal{O}_{\ell^{(m)}}(-2 j), n\right)+2 \phi\left(\mathcal{O}_{\ell^{(m)}}, n\right)+\phi\left(\mathcal{O}_{\ell^{(m)}}(2 j), n\right) .
\end{aligned}
$$

We conclude this proof by computing $H^{0}\left(\ell^{(m)} ; \mathcal{O}(p)\right)$. Now we have to consider the spaces $Z_{k}$ and $W_{1}$ separately. We pick a chart $U$ with local coordinates $(z, u)$ on $Z_{k}$ and $(z, u, v)$ on $W_{1}$, respectively, which transform to $\left(z^{-1}, z^{k} u\right)$ and $\left(z^{-1}, z u, z v\right)$.

On $\ell^{(m)} \subset Z_{k}$, a section $a \in \mathcal{O}(p)(U)$ is a function $a(z, u)=\sum_{r=0}^{m} \sum_{s=0}^{\infty} a_{r s} z^{s} u^{r}$ such that $\sum_{r, s} a_{r s} z^{s-p} u^{r}$ is holomorphic in $\left(z^{-1}, z^{k} u\right)$, i.e. $s-p \leq k r$. Thus

$$
a(z, u)=\sum_{r=0}^{m} \sum_{s=0}^{k r+p} a_{r s} z^{s} u^{r}
$$

which has $\frac{1}{2}(m+1)(k m+2+2 p)=: \phi_{\mathcal{O}}(p)$ coefficients.

On $\ell^{(m)} \subset W_{1}$, a section $a \in \mathcal{O}(p)(U)$ is $a(z, u, v)=\sum_{t=0}^{m} \sum_{r=0}^{m-t} \sum_{s=0}^{\infty} a_{t r s} z^{s} u^{r} v^{t}$ such that $\sum_{t, r, s} a_{t r s} z^{s-p} u^{r} v^{t}$ is holomorphic in $\left(z^{-1}, z u, z v\right)$, i.e. $s-p \leq r+t$. Thus

$$
a(z, u, v)=\sum_{t=0}^{m} \sum_{r=0}^{m-t} \sum_{s=0}^{r+t+p} a_{t r s} z^{s} u^{r} v^{t},
$$

which has $\frac{1}{6}(m+2)(m+1)(2 m+3 p+3)=: \phi(\mathcal{O}, p)$ coefficients.

Putting it all together, we have

$$
\begin{aligned}
\phi\left(E^{(m)}, n\right) & =\phi(\mathcal{O},-j+n)+\phi(\mathcal{O}, j+n), \\
\phi\left(\mathcal{E} n d E^{(m)}, n\right) & =\phi(\mathcal{O},-2 j+n)+2 \phi(\mathcal{O}, n)+\phi(\mathcal{O}, 2 j+n),
\end{aligned}
$$

which gives the desired functions.

3.1. Examples of invariants. To make the notion of the numbers we defined above more concrete, we tabulate examples for the two bundles $E=\mathcal{O}(-3) \oplus \mathcal{O}(3)$ (the split bundle of splitting type 3 ) and $G$, the "most generic" bundle of splitting type 3 (which has the lowest invariants among all bundles of splitting type 3 ), on the spaces $Z_{1}, Z_{2}, Z_{3}$ and $W_{1}$; see Table 1 . 


\section{SURFACES}

Let $Z_{k}:=\operatorname{Tot}\left(\mathcal{O}_{\mathbb{P}^{1}}(-k)\right)$ and let $E$ be a rank-2 bundle on $Z_{k}$ with $c_{1}(E)=0$ and splitting type $j$. Then $E$ is determined by an element $p \in \operatorname{Ext}^{1}(\mathcal{O}(j), \mathcal{O}(-j))$ as in (3.4). The direct image $\pi_{*}(E)$ is a reflexive sheaf on $X_{k}$, and there are bounds for its local holomorphic Euler characteristic around the singular point $x \in X_{k}$ in terms of $j$. An efficient algorithm to compute $\mathbf{w}, \mathbf{h}$ and $\chi$ is given in http://www.maths.ed.ac.uk/ s0571100/Instanton/, hence we can explicitly calculate the values of these numerical invariants for any such bundle $E$. We present here a useful existence result.

Lemma 4.1. Let $E$ be a rank-2 bundle over $Z_{k}, k>1$, with $c_{1}(E)=0$ and splitting type $j<k$. Then

$$
\chi\left(x, \pi_{*} E\right)=j-1 .
$$

Proof. By [G, Theorem 3.3] it follows that if $j<k$ then $E \cong \mathcal{O}_{Z_{k}}(j) \oplus \mathcal{O}_{Z_{k}}(-j)$. By definition, $\chi\left(x, \pi_{*} E\right)=\mathbf{w}(E)+\mathbf{h}(E)$. Direct computation (see [BGK1]) then shows that $\mathbf{w}(E)=0$ and $\mathbf{h}(E)=j-1$.

In fact, we can say a lot more.

Lemma 4.2. [BGK1, Corollary 2.18] Let $E$ be a rank-2 bundle over $Z_{k}, k>1$, with splitting type $j>0$. Set $j=q k+r$ with $0 \leq r<q$. The following bounds are sharp:

$$
j-1 \leq \chi\left(x, \pi_{*} E\right) \leq \begin{cases}q^{2} k+(2 q+1) r-1 & \text { if } 1 \leq r<k, \\ q^{2} k & \text { if } r=0 .\end{cases}
$$

Remark 4.3. Note that every bundle that satisfies the conditions of Lemma 4.1 is split, whereas in general there are many distinct isomorphism classes of bundles, which attain a whole range of numerical invariants. The lower bound in Lemma 4.2 is attained by a class of generic bundles, while the upper bound is obtained by the split bundle of splitting type $j$, and moreover, the split bundle is the only bundle to attain the bound when $r=0$.

These two lemmas directly imply the following existence result.

Theorem 4.4. Let $\mathfrak{M}_{n}\left(X_{k}\right)$ be the moduli of reflexive sheaves on $X_{k}$ with local holomorphic Euler characteristic equal to $n$. Then for all $n \geq 0, \mathfrak{M}_{n}\left(X_{k}\right)$ is non-empty.

4.1. Applications to physics. To illustrate applications to physics, we mention some results on the existence of instantons. We stress that this particular instance of gaps on instantons charges presented below was completely new to physicists. In fact, there was a folklore belief that 1-instantons are always the most common, and that higher instantons of charge $k$ should decay to $k$ instantons of charge 1 over time. Our results showed that over the spaces $Z_{k}$ with $k \geq 3$ there do not exist any 1-instantons, nevertheless higher charge instantons do exist (of course we mean mathematical existence proofs).

In [GKM, Proposition 54] we studied the Kobayashi-Hitchin correspondence for the spaces $Z_{k}$ : We showed that an $S U(2)$-instanton on $Z_{k}$ of charge $n$ corresponds to a holomorphic $S L(2)$ bundle $E$ on $Z_{k}$ with $\chi(\ell, E)=n$ together with a trivialization of $\left.E\right|_{Z_{k}^{\circ}}$, where $Z_{k}^{\circ}:=Z_{k}-\ell$. A simple observation [GKM, Proposition 4.1] shows that there exists a trivialization of $\left.E\right|_{Z_{k}^{\circ}}$ if and only if $n=0 \bmod k$. This restricts the splitting type of an instanton bundle over $Z_{k}$ to be of the form $n k$ and lead us to the following existence/non-existence result:

Proposition 4.5. [GKM, Theorem 6.8] The minimal local charge of a non-trivial SU(2)instanton on $Z_{k}$ is $\chi_{k}^{\min }=k-1$. The local moduli space of (unframed) instantons on $Z_{k}$ with fixed local charge $\chi_{k}^{\min }$ has dimension $k-2$. 
This result shows a straightforward passage from the algebraic geometry of bundles on surfaces to meaningful mathematical physics. Similar results for Calabi-Yau threefolds promise to have exciting interpretations in string theory and physics, whenever the mathematical background is constructed.

Remark 4.6. (Gaps of instanton charges) The non-existence of instantons with certain local charges on the spaces $Z_{k}$ for $k>2$ is in stark contrast with what happens in the case $k=1$, where there is no gap [BG, Theorem 0.2].

Open Question 4.7. Theorem 4.2 gives sharp bounds for $\chi$-are the intermediate values achieved? Given an integer $\alpha$ such that $j-1<\alpha<q^{2} k$, does there exist an instanton bundle on $Z_{k}$ with splitting type $j$ and $\chi=\alpha$ ? We have a positive answer for analogous question when $k=1$, all other cases are open.

We illustrate also an application to topology:

Theorem 4.8. [BGK1, Theorem 4.15] If $j=q k$ for some $q \in \mathbb{N}$, then the pair $(\mathbf{w}, \mathbf{h})$ stratifies instanton moduli stacks $\mathfrak{M}_{j, k}$ into Hausdorff components.

Open Question 4.9. Find invariants that stratify the moduli stacks $\mathfrak{M}_{j, k}$ in the case $j=n k+r$ with $r \neq 0 \bmod k$. We know that the pair $(\mathbf{w}, \mathbf{h})$ does not provide a fine enough invariant to stratify the moduli stacks in these cases. Thus, some extra numerical invariant is needed. At the moment the authors are completely unaware of any suitable candidate.

We find it completely surprising that the case $r=0$, whose physics interpretation is known, turned out to be much simpler to solve. From a topological point of view one should of course have Hausdorff stratifications for the moduli stacks in all cases.

\section{ThreEFolds}

Consider a smooth threefold $W$ containing a line $\ell \cong \mathbb{P}^{1}$. We will focus on the Calabi-Yau cases

$$
W_{i}:=\operatorname{Tot}\left(\mathcal{O}_{\mathbb{P}^{1}}(-i) \oplus \mathcal{O}_{\mathbb{P}^{1}}(i-2) \text { for } i=1,2,3 .\right.
$$

The existence of a contraction of $\ell$ imposes heavy restrictions on the normal bundle [Jim], namely $N_{\ell / W}$ must be isomorphic to one of
(a) $\mathcal{O}_{\mathbb{P}^{1}}(-1) \oplus \mathcal{O}_{\mathbb{P}^{1}}(-1)$,
(b) $\mathcal{O}_{\mathbb{P}^{1}}(-2) \oplus \mathcal{O}_{\mathbb{P}^{1}}(0)$, or
(c) $\mathcal{O}_{\mathbb{P}^{1}}(-3) \oplus \mathcal{O}_{\mathbb{P}^{1}}(+1)$.

Conversely, Jiménez states that if $\mathbb{P}^{1} \cong \ell \subset W$ is any subspace of a smooth threefold $W$ such that $N_{\ell / W}$ is isomorphic to one of the above, then:

- in (a) $\ell$ always contracts,

- in (b) either $\ell$ contracts or it moves, and

- in case (c) there exists an example in which $\ell$ does not contract nor does any multiple (i.e. any scheme supported on $\ell$ ) move.

$W_{1}$ is the space appearing in the basic flop. Let $X$ be the cone over the ordinary double point defined by the equation $x y-z w=0$ on $\mathbb{C}^{4}$. The basic flop is described by the diagram:

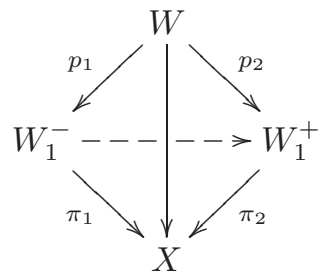


Here $W:=W_{x, y, z, w}$ is the blow-up of $X$ at the vertex $x=y=z=w=0, W_{1}^{-}:=Z_{x, z}$ is the small blow-up of $X$ along $x=z=0$ and $W_{1}^{+}:=Z_{y, w}$ is the small blow-up of $X$ along $y=w=0$. The basic flop is the rational map from $W^{-}$to $W^{+}$.

In $W_{2} \cong Z_{2} \times \mathbb{C}$ the zero section does not contract to a point (so it must be able to move), but it is possible to contract it partially and obtain a singular family $X_{2} \times \mathbb{C}$, where $X_{2}$ is the surface containing an ordinary double-point singularity defined by $x y-z^{2}=0$ in $\mathbb{C}^{3}$. Holomorphic bundles on $W_{2}$ have infinite local holomorphic Euler characteristic, but the restriction $\left.E\right|_{Z_{2} \times\{0\}}$ has well-defined and finite width and height. Note that in contrast to $W_{1}$, there are strictly holomorphic (non-algebraic) bundles on $W_{2}$, although every rank-2 bundle on $W_{2}$ is still an extension of line bundles.

In $W_{3}$ not even a partial contraction of the zero section is possible. Nevertheless we can still calculate the width and height of the restriction $\left.E\right|_{Z_{3}}$ of a bundle $E$ to a subsurface $Z_{3} \hookrightarrow W_{3}$. Again, on $W_{3}$ there are strictly holomorphic (non-algebraic) bundles, and moreover, there are (many) rank-2 bundles which are not extensions of line bundles.

5.1. Bounds and generating functions. We can compute the invariants $\mathbf{w}(E), \mathbf{h}(E)$ and $h^{1}(\mathcal{E} n d E)$ directly and algorithmically. We have an implementation of each of the algorithms for the commutative algebra software Macaulay 2, which led us to discover several formulae for the bounds of these invariants. Bounds for the local holomorphic Euler characteristic $\chi=\mathbf{w}+\mathbf{h}$ on surfaces were presented in Section 4; now we turn to the flop space $W_{1}$, were by Lemma 3.3, we have $\chi=\mathbf{h}$.

Theorem 5.1. For every rank-2 bundle $E$ on $W_{1}$ with $c_{1}(E)=0$ and splitting type $j$, the following bounds are sharp:

$$
j-1 \leq \chi(\ell, E)=\mathbf{h}(E) \leq\left(j^{2}+j\right)(j-1) / 6 .
$$

Proof. The lower bound is attained by a class of generic bundles, and the upper bound by the split bundle $\mathcal{O}(-j) \oplus \mathcal{O}(j)$. This can be seen by direct computation as explained in [BGK1] and $[\mathrm{Kö}]$.

We also have a concise expression for the numbers $h^{1}(\mathcal{E} n d)$ of the extremal cases, that is generic and the split bundles of splitting type $j$.

Definition 5.2. A power series of the form $g(z)=\sum_{j=0}^{\infty} a_{j} z^{j}$ is called a generating function for the sequence $\left(a_{j}\right)_{j=0}^{\infty}$. Hence, $a_{j}=\left.\frac{1}{j !} \frac{d^{j} g}{d z^{j}}\right|_{z=0}$.

Set $a_{j}^{X, E}:=h^{1}(X ; \mathcal{E} n d E)$. Then if the base space is $X=Z_{k}$ or $W_{1}$ and the bundle $E$ over $X$ is either split or generic of splitting type $j$, we have generating functions for $a_{j}^{X, E}$, as shown in Table 2. Since the generating function of a sum of two sequences is the sum of the generating functions, we can easily deduce from this the generating functions for $\Delta_{0}$ and $\Delta_{1}$. We spell out the inequalities.

Theorem 5.3. For every rank-2 bundle $E$ on $W_{1}$ with $c_{1}(E)=0$ and splitting type $j$, the following bounds are sharp:

$$
\left(j^{3}+3 j^{2}-j\right) / 3 \leq h^{1}\left(W_{1} ; \mathcal{E} n d E\right) \leq\left(4 j^{3}-j\right) / 3
$$

Proof. The lower bound is attained by a generic bundle and the upper bound by the split bundle, the values are found by direct computation. 


\begin{tabular}{ccc}
\hline Space & Split bundle $E_{j}$ & Generic bundle $G_{j}$ \\
\hline$Z_{k}, k=2 n$ & $\frac{-z\left(z^{n+1}+z^{n}+z+1\right)}{(z-1)^{2}\left(z^{k}-1\right)}$ & $\frac{z^{k+2}-z^{3}-z^{2}-z}{(z-1)^{2}\left(z^{k}-1\right)}$ \\
$Z_{k}, k=2 n+1$ & $\frac{-z\left(2 z^{n+1}+z+1\right)}{(z-1)^{2}\left(z^{k}-1\right)}$ & \\
$W_{1}$ & $\frac{z\left(z^{2}+6 z+1\right)}{(z-1)^{4}}$ & $\frac{z\left(-z^{2}+2 z+1\right)}{(z-1)^{4}}$ \\
\hline
\end{tabular}

TABLE 2. Generating functions for $a_{j}^{X, E}:=h^{1}(X ; \mathcal{E} n d E)$ on various spaces for the split and the generic bundle of splitting type $j$ (data for $G_{j}$ only valid for $j \geq k$ ); the value $a_{j}^{X, E}$ is the $j^{\text {th }}$ coefficient in the Taylor series.

5.2. Moduli of sheaves. We consider sheaves on singular varieties obtained as direct images of bundles on $W_{i}$. First we study such bundles and their moduli. The topological structure of these moduli is not yet well understood. Most numerical invariants defined in Section 3 can be computed over any $W_{i}$; however, the invariants $\Delta_{0}$ and $\Delta_{1}$ in (3.5) are infinite on $W_{2}$ and $W_{3}$, so more refined counterparts are required.

Open Question 5.4. Construct a Hausdorff stratification of the moduli stacks $\mathfrak{M}_{n}\left(W_{i}\right)$ of bundles on $W_{i}$ with $c_{1}=0$ and $\chi(\ell, E)=n$.

We obtain a partial understanding of these moduli by looking at first-order deformations, and this will provide enough bundles for an existence theorem of reflexive sheaves on the corresponding singular varieties.

Proposition 5.5. (First-order deformations) Set $F:=\mathcal{O}_{\ell}(-j) \oplus \mathcal{O}_{\ell}(j)$ with $\ell \subset W_{i}$.

(1) For any bundle $E$ on $W_{i}$ with $\left.E\right|_{\ell} \cong F$, the space of first-order deformations of $G$ is isomorphic to $\mathbb{C}^{\gamma_{1}}$, where

$$
\gamma_{1}:=h^{1}\left(\ell ; \mathcal{E} n d\left(\left.E\right|_{\ell}\right) \otimes \mathcal{I}_{\ell} / \mathcal{I}_{\ell}^{2}\right)<\infty .
$$

(2) If $\mathcal{I}_{\ell} / \mathcal{I}_{\ell}^{2}$ is ample (i.e. if $i=1$ ), then there exists a vector bundle $A$ on $W_{1}$ such that $\left.A\right|_{\ell} \cong F$.

Proof. The dimension count is standard deformation theory. Existence of extensions to formal and small analytic neighbourhoods of $\ell$ are given by Peternell's Existence Theorem [Pet]. The fact that we actually get existence on the entire space $W_{1}$ rather than just a small neighbourhood of $\ell$ is due to the fact that every bundle on $W_{1}$ is determined by its restriction to a finite infinitesimal neighborhood of $\ell$.

Corollary 5.6. (Dimension of moduli) The moduli space of first order deformations of $\mathcal{O}(j) \oplus$ $\mathcal{O}(-j)$ over $W_{i}$ modulo holomorphic isomorphisms is isomorphic to $\mathbb{P}^{4 j-5}$.

Proof. It is well known that multiplying the extension class by a non-zero constant does not change the holomorphic type of the underlying bundle. It turns out that on the first formal 
neighborhood this is the only identification. This was proved for surfaces in [BGK1, Theorem 4.9] and for $W_{i}, i=1,2,3$ in [Kö]. We can then compute $\gamma_{1}$ directly as the dimension of the first cohomology of $\mathcal{E} n d\left(\mathcal{O}_{\mathbb{P}^{1}}(-j) \oplus \mathcal{O}_{\mathbb{P}^{1}}(j)\right) \otimes N_{\ell / W_{i}}^{*}$ on $\mathbb{P}^{1}$. The $\mathcal{E} n d$-bundle splits into a direct sum of line bundles, and the computation is straightforward.

If instead of the first-order deformations we wish to consider all deformations, then the dimension of the deformation space is given by

$$
\gamma:=\sum_{m=0}^{\infty} h^{1}\left(\ell ; \mathcal{E} n d\left(\left.E\right|_{\ell}\right) \otimes \operatorname{Sym}^{m}\left(\mathcal{I}_{\ell} / \mathcal{I}_{\ell}^{2}\right)\right)
$$

which is finite when $\mathcal{I}_{\ell} / \mathcal{I}_{\ell}^{2}$ is ample, but infinite in general. Though the space of deformations may be infinite, it turns out that for a fixed $j$ the moduli space $\mathfrak{M}_{n}\left(W_{i}\right)$ of holomorphic bundles $E$ on $W_{i}$ with $\chi(\ell, E)=n=j-1$ has a Zariski-open set of dimension $4 j-5$ consisting of of first-order deformations of $\mathcal{O}(j) \oplus \mathcal{O}(-j)$ (cf. Corollary 5.6). Now, using these moduli for the case of $W_{1}$, we obtain sheaves on the singular threefold $X$ appearing on the flop diagram (5.1).

Theorem 5.7. Let $X$ be the singular threefold $x y-z w=0$ in $\mathbb{C}^{4}$. For each $j \geq 2$ there exists $a(4 j-5)$-dimensional family of rank-2 reflexive sheaves on $X$ with local holomorphic Euler characteristic $j-1$.

Proof. These reflexive sheaves are obtained as direct images of generic bundles on $W_{1}$ with splitting type $(-j, j)$. Combine Corollary 5.6 with the value of $\chi$ found for the generic bundle as given in Table 2.

For the case of $j=0$ or 1 our methods give only the direct images of the split bundles $\mathcal{O} \oplus \mathcal{O}$ and $\mathcal{O}(1) \oplus \mathcal{O}(-1)$, both have $\chi=0$.

We stop short of stating a similar theorem for the singular spaces obtained by partial contractions on $W_{i}$ with $i=2,3$ because strictly speaking the definition of local Euler characteristic was given for isolated singularities. We do obtain existence of reflexive sheaves on those spaces, but we do not yet have a good feel for what would be the correct numerical numerical invariants to use.

Open Question 5.8. Describe the full moduli of reflexive rank-2 sheaves on $W_{1}$ with $c_{1}=0$ and $\chi=n$, that is, include all sheaves that do not occur as direct images of bundles on $W_{1}$.

Open Question 5.9. Describe moduli of sheaves with fixed numerical invariants on germs of singularities.

The latter is of course a very big question, actually infinitely many open questions, starting with the definition of the correct invariants up to their computation and then construction of moduli. It is certainly an entire research project for a whole group of singularists. We hope some singularists get inspired to work on these questions.

\section{REFERENCES}

[BG] E. Ballico, E. Gasparim, Vector bundles on a neighborhood of a curve in a surface and elementary transformations, Forum Math. 15 (2003), no. 1, 115-122.

[BGK1] E. Ballico, E. Gasparim, T. Köppe, Vector bundles near negative curves: moduli and local Euler characteristic, Comm. Alg. 37 (2009), no. 8, 2688-2713.

[BGK2] E. Ballico, E. Gasparim, T. Köppe, Local moduli of holomorphic bundles, J. Pure Appl. Algebra 213 (2009), 397-408.

[Bl] R. Blache, Chern classes and Hirzebruch-Riemann-Roch theorem for coherent sheaves on complexprojective orbifolds with isolated singularities, Math. Z. 222 (1996), no. 1, 7-57. 
[Br] J.-P. Brasselet, From Chern classes to Milnor classes - a history of characteristic classes for singular varieties, Adv. Stud. Pure Math. 29 (2000), 31-52.

[G] E. Gasparim, Holomorphic bundles on $\mathcal{O}(-k)$ are algebraic, Comm. Algebra 25 (1997), no. 9, 30013009.

[GKM] E. Gasparim, T. Köppe, P. Majumdar, Local holomorphic Euler characteristic and instanton decay, Pure Appl. Math. Q. 4 (2008), no. 2, 161-179, Special Issue: In honor of Fedya Bogomolov, Part 1.

[GL] E. Gasparim, C. M. Liu, The Nekrasov conjecture for toric surfaces, Comm. Math. Phys. 293 (2010), no. $3,661-700$.

[Jim] J. Jiménez, Contraction of nonsingular curves, Duke Math. J. 65 (1992), no. 2, 313-332.

[Kö] T. Köppe, Ph. D. thesis, University of Edinburgh (2010).

[MNOP] D. Maulik, N. Nekrasov, A. Okounkov, R. Pandharipande, Gromov-Witten theory and DonaldsonThomas theory I., Compos. Math. 142 (2006), no. 5, 1263-1285.

[Pet] T. Peternell, Vektorraumbündel in der Nähe von exzeptionellen Unterräumen - das Modulproblem, J. Reine Angew. Math. 336 (1982), 110-123.

School of Mathematics, The University of Edinburgh, James Clerk Maxwell Building, The King's Buildings, Mayfield Road, Edinburgh, EH9 3JZ, United Kingdom

E-mail address: Elizabeth.Gasparim@ed.ac.uk

E-mail address: t.koeppe@ed.ac.uk 